\title{
Die Anpassung diskriminierender Tarifverträge an das Recht
}

\section{Prof. Dr. Eva Kocher}

Mitglied der Kommission Arbeits-, Gleichstellungs- und Wirtschaftsrecht des djb, Europa-Universität Viadrina, Frankfurt/ Oder

\section{Gender Pay Gap und Regelungsbedarf - Schwäche freiwilli- ger Instrumente}

Die verfassungs- und europarechtliche Garantie „gleiches Entgelt für Männer und Frauen bei gleicher oder gleichwertiger Arbeit “ (Art. 3 II GG, Art. 157 AEUV1) wird in der Rechtswirklichkeit nicht eingelöst: Überall in Europa verdienen Frauen weniger als Männer. Der Gender Pay Gap, also der prozentuale Unterschied im durchschnittlichen Bruttostundenverdienst von Frauen und Männern, liegt in Deutschland derzeit über 23 Prozent. 2007 lag Deutschland damit auf Platz 21 der 27 EU-Mitgliedstaaten. ${ }^{2}$

Auch Bundesministerin Kristina Schröder hat mittlerweile erkannt, dass die Entgeltungleichheit zwar viele Ursachen hat, diskriminierende betriebliche (Entgelt-)Strukturen aber einen wichtigen Teil dazu beitragen. ${ }^{3}$ Sie bietet deshalb den Unternehmen das „kostenlose Beratungspaket“ an, damit diese selbst und freiwillig herausfinden, ob Entgeltunterschiede im Unternehmen bestehen, die auf das Geschlecht zurückzuführen sind. ${ }^{4}$ Der djb kritisiert das „Selbsttestinstrument“ Logib$\mathrm{D}$, weil es den Unternehmen von vornherein nicht die erforderlichen Informationen liefert, die zur Aufdeckung von Geschlechtsdiskriminierung beim Entgelt geeignet wären. Ein Unternehmen, das eine solche Analyse wünscht, signalisiert ja bereits Problembewusstsein; ein geeignetes Instrument müsste also in der Lage sein, wahrscheinliche Problembereiche anzudeuten, anstatt Unternehmen trügerisch in Sicherheit zu wiegen. Aber ganz unabhängig von dieser Kritik am konkreten Instrument: Es ist schon lange erwiesen, dass freiwillige Instrumente im Bereich der Gleichstellung im Erwerbsleben auf breiter Ebene wenig ausrichten können. Wie erwartet hat auch der Verzicht auf ein Gesetz zugunsten der „Vereinbarung zwischen der Bundesregierung und den Spitzenverbänden der deutschen Wirtschaft zur Förderung der Chancengleichheit von Frauen und Männern in der Privatwirtschaft" aus dem Jahr 2001 an den Geschlechterverhältnissen in der Privatwirtschaft nichts Sichtbares geändert ${ }^{5}$.

Dazu kommt: Entgeltdiskriminierung geht häufig auf kollektive Entgeltsysteme zurück, die tariflich geregelt sind. Der individuelle Rechtsanspruch ,gleiches Entgelt für gleichwertige Arbeit" ist aber in seiner Grundstruktur auf individuelle Vergleiche angelegt. Eine Frau, die gleiches Entgelt einklagt, kann sich aussuchen, mit wem sie sich vergleicht. Der Arbeitgeber muss/kann dann einen sachlichen Grund für die Differenzierung nennen. ${ }^{6}$ Allerdings kann die Vergleichsperson nicht willkürlich gewählt werden; es muss eine Person sein, die „gleichwertige“ Arbeit ausführt. Und damit sind wir bei der Problematik der Arbeitsbewertung, die hinter und über den kollektiven Entgeltsystemen liegt. Denn die Arbeit einer einzelnen Person kann nicht isoliert bewertet werden; jede Bewertung bezieht sich auf Vergleich und damit auf übergeordnete Werte- und Bewertungssysteme. In der Regel hängen die Arbeits- und Tätigkeitsbewertungen aller Beschäftigten deshalb eng miteinander zusammen.

Um den Entgeltgleichheitsanspruch wirksam machen zu können, bedarf es einer Arbeitsbewertung nach geschlechtergerechten Grundsätzen und Analysen, mit der Gleichwertigkeit dargestellt werden könnte. Dies ist durchaus komplex und schwierig7. Dafür müssten jeweils ganze Entgeltsysteme in ihrer Gesamtstruktur überprüft werden. In Bezug auf Tarifverträge gibt und gab es dafür schon eine Reihe von Pilotprojekten. Weil aber auch diese angesichts der Mitgliederstruktur und des Interessengegensatzes zwischen den Tarifparteien nicht die erhoffte Verbreiterung gebracht haben ${ }^{8}$, wird als rechtspolitischer Vorschlag für eine effektive Durchsetzung der Entgeltgleichheit immer wieder die Forderung nach einem Verfahrensgesetz erhoben, das die tariflichen und betrieblichen Akteurinnen und Akteure wirksam in die Pflicht nehmen könnte.

\section{Ein Verfahrensgesetz für „Pay Equity Plans“}

Sibylle Raasch hat deshalb vor Kurzem in Anlehnung an Heide Pfarr ${ }^{9}$ in der djbZ unter anderem ${ }^{10}$ vorgeschlagen ${ }^{11}$, dass bestehende Tarifverträge innerhalb gesetzlich festzulegender Fristen nachträglich einer Überprüfung auf Diskriminierung durch die daran beteiligten Tarifvertragsparteien unterzogen werden sollten. Hierfür dient wiederum ein Verfahrensgesetz aus der kanadischen Provinz Ontario als Vorbild, das sich nach seinem Erlass im Jahre 1987 als immerhin zureichend erfolgreich erwiesen hat. ${ }^{12}$ Es verpflichtet Arbeitgeber und/oder Sozial-/Tarifparteien gesetzlich dazu, Lohngleich-

\footnotetext{
1 Ex-Art. 141 EGV.

2 Z.B. Kommissionsbericht 2008 über die Gleichstellung von Frauen und Männern, $\operatorname{KOM(2008)10~endg.,~} 13$.

3 Siehe schon Bericht der Bundesregierung zur Berufs- und Einkommenssituation von Frauen und Männern, vom 25.4.2002, BTDrucks. $14 / 8952$

4 Siehe <http://www.logib-d.de> (Zugriff 19.7.2010).

5 Zur Kritik siehe schon Raasch, Gender Pay Gap mit neuen Instrumenten überwinden, djbZ 2009, 126. Zur Vereinbarung siehe den Text auf <http://www.dihk.de/inhalt/download/chancengleichheit.pdf> sowie Berichte auf <http://www.genderkompetenz.info/ genderkompetenz-2003-2009/sachgebiete/wirtschaft/aspekte> (Zugriff jeweils 19.7.2010).

6 Ausführlich Colneric, Der Anspruch auf gleichen Lohn für gleichwertige Arbeit und das Verbot der mittelbaren Diskriminierung, Festschrift für Dieterich 1999, $45 \mathrm{ff}$.

7 Zum Problem vgl. Schiek/M. Schmidt, AGG-Kommentar, 2006, § 8, Rn. 6. Siehe auch Art. 4 Abs. 2 der Richtlinie 2006/54/EG.
} 
heitskommissionen einzusetzen, deren Aufgabe darin besteht, kollektive Entgeltsysteme auf Diskriminierungselemente zu überprüfen und sie geschlechtergerecht umzugestalten. Die Tarifvertragsparteien hätten es auf diese Art und Weise zunächst selbst in der Hand, die interne Ausgewogenheit des geschlechtergerechten Entgeltsystems zu verhandeln. Käme es nicht zu einer Einigung, so wäre eine Schiedsstelle („Entgeltgleichheitskommission“) unter unabhängigem Vorsitz einzusetzen, die über das weitere Vorgehen zu entscheiden hätte.

Damit würden die Tarifverhandlungen und die Tarifautonomie nur verfahrensmäßig gestaltet, inhaltlich aber nicht stärker beeinflusst, als es das Entgeltgleichheitsgebot ohnehin erfordert. Es besteht ja kein Zweifel daran, dass die Tarifparteien sich eine rechtliche Überprüfung der Tarifverträge auf die Einhaltung des Entgeltgleichheitsgebots gefallen lassen müssen. Diskriminierende Kollektivverträge widersprechen den zahlreichen Rechtsvorschriften zur Entgeltgleichheit. Für die Mitgliedstaaten der EU ist Entgeltgleichheit unabhängig vom Geschlecht mit unmittelbarer Wirkung in Art. 157 AEUV geregelt. Die Vorschrift wird konkretisiert durch die Richtlinie 2006/54/EG. Für das deutsche Recht erklärt $\ 7$ Abs. 2 AGG Bestimmungen in Vereinbarungen, die dem Diskriminierungsverbot widersprechen, ausdrücklich für unwirksam; dies gilt auch für Kollektivvereinbarungen wie Tarifverträge. ${ }^{13}$ Die Gerichte müssen diskriminierende Tarifnormen überprüfen und unangewendet lassen. ${ }^{14}$ Die ex-post-Kontrolle verfolgt damit das Ziel, Tarifverträge nicht etwa inhaltlich weitergehend $\mathrm{zu}$ kontrollieren als bisher, sondern vielmehr die Kontrolle effektiver zu machen.

\section{Schrittweise Anpassung}

Das Problem bei den nachträglichen Überprüfungen liegt woanders: Es gibt in der Regel nicht nur eine einzige Möglichkeit, um Entgeltgleichheit für die Zukunft herzustellen, sondern viele unterschiedliche Varianten; wenn statt der diskriminierenden Bewertungsmerkmale nun geschlechtergerechte Eingruppierungskriterien verwendet werden, kann und soll sich ja die Gewichtung insgesamt verändern. Deshalb muss die Überprüfung des Systems als Ganzem auch den Tarifparteien überlassen bleiben und kann nicht einer Behörde oder einem Gericht übertragen werden. Wie kommt man aber sinnvollerweise von einem Entgeltsystem zu einem anderen, nicht diskriminierenden?

Es ist gar nicht so einfach, von einem Tag auf den anderen auf ein neues Entgeltsystem umzustellen. Zum einen gibt es das rechtliche Problem, dass die bestehenden Besitzstände und Ansprüche der bislang bevorzugten (männlichen) Beschäftigten in der Regel zumindest individualvertraglich abgesichert sind und nicht einfach gekürzt werden können. Andererseits wäre eine Erhöhung der Lohnsumme auch faktisch-politischwirtschaftlich äußerst problematisch: Sie wäre jedenfalls ein starkes wirtschaftliches Argument für eine ohnehin zu befürchtende „mächtige Koalition zwischen den bislang begünstigten Männern und der Unternehmensseite zur Verhinderung grundlegender Veränderungen “. ${ }^{15}$ Sollen auf breiter Ebene Ef- fekte erzielt werden, so kommt wohl nur eine schrittweise Anpassung in Stufen in Betracht. Diese Anpassung ist Gegenstand dessen, was man den „Pay Equity Plan“ nennt: ein Entgelt-Anpassungsplan, der beschreibt, welche Entgeltlücken bestehen, und der regelt, wie bzw. innerhalb welcher Fristen sie ausgeglichen werden.

\section{Bedeutung für Individualansprüche auf Entgeltgleichheit}

Für die Individualansprüche der bislang benachteiligten Frauen bedeutet dies: Das Entgelt würde erst nach und nach angehoben; der Entgeltunterschied würde nach und nach abgeschmolzen. ${ }^{16}$ Das bedeutet aber auch: Diskriminierungswirkungen aus der früheren Regelung wirken zunächst fort.

Falls im Einzelfall eine Diskriminierung festgestellt wird, fordert der Gleichbehandlungsanspruch aber, dass die Entgelte der bislang benachteiligten (weiblichen) Beschäftigten angehoben werden, um Entgeltgleichheit herzustellen - jedenfalls solange kein anderes (kollektives) diskriminierungsfreies System hergestellt ist. ${ }^{17}$ Während der Anpassungsphase des Pay Equity Plans bestünden die Individualansprüche auf Entgeltgleichheit also fort, der Plan wäre notwendig rechtswidrig. Pfarr hatte deshalb vorgeschlagen, dass die individuellen Ansprüche sich nach den Ergebnissen des kollektiven Verhandlungsverfahrens richten sollten. Individualrechtliche Entgeltgleichheitsklagen sollten ausgesetzt werden, damit und soweit im Betrieb des beklagten Arbeitgebers eine Überprüfung des Entgeltsystems auf Diskriminierungsfreiheit stattfindet; das Ergebnis der Überprüfung, der Pay Equity Plan, solle dann Rechtsgrundlage der Entscheidung des Arbeitsgerichts werden.

\section{Die rechtliche Zulässigkeit der schrittweisen Anpassung}

Wenn eine solche schrittweise Anpassung aus Sicht der Geschlechtergleichbehandlung rechtspolitisch angemessen und sinnvoll erscheint, muss es umso mehr enttäuschen, wenn ge-

8 Pfarr, Entgeltgleichheit in kollektiven Entgeltsystemen, Festschrift 50 Jahre BAG, 2004, 779 ff. (788).

9 Siehe Fn. 8; <http://www.boeckler.de/pdf/wsi pfarr gesetzentwurf_entgeltgleichheit.pdf> (Zugriff 19.7.2010). Der Entwurf ergänzt einen breiteren Entwurf der Expertinnenkommission für ein Gleichstellungsgesetz für die Privatwirtschaft (Pfarr (Hrsg.), Ein Gesetz zur Gleichstellung der Geschlechter in der Privatwirtschaft, 2001; Laskowski, ZRP 2001, 504 ff.) um die Probleme der Durchsetzung von Entgeltgleichheit.

10 Auf die ebenfalls vorgeschlagene ex-ante-Überprüfung gehe ich in diesem Text nicht ein; sie wirft noch einmal weitergehende Fragen der Tarifautonomie auf.

11 Raasch (Fn. 5).

12 Winter, Gleiches Entgelt für gleichwertige Arbeit, 1998, 315 ff.; 341 ff.; 359 ff.; zur Effektivität siehe Pfarr (Fn. 8), 788, Fn. 69.

13 Siehe zum Beispiel Schiek/M. Schmidt, (Fn. 7), \$ 7, Rn. 3.

14 Siehe jetzt wieder BAG 20.5.2010 - 6 AZR 148/og (A); BAG 20.5.2010 - 6 AZR 319/og (A); das BAG verweist aus der Rechtsprechung des EuGH auf EuGH 7.2.1991 - C-184/89 (Nimz); EuGH 27.6.1990 - C-33/89 (Kowalska).

15 Raasch (Fn. 5).

16 Pfarr (Fn. 8); dazu bereits Kocher, KJ 2007, 22 ff.; Kocher, Individualansprüche als Bremse proaktiver Politiken der Entgeltgleichheit? in: Arioli u.a. (Hrsg.): Wandel der Geschlechterverhältnisse durch Recht?, 2008, 135 ff.; zum Ontario-Gesetz Winter, (Fn. 12); anders Schiek/Schiek, AGG, Einl, Rn. 58. 
rade der gemeinschaftsrechtliche Grundsatz der Entgeltgleichheit entgegenstehen soll. ${ }^{18}$ Richtig ist, dass Art. 157 AEUV einen unbedingten Individualanspruch auf Entgeltgleichheit enthält. Dies lässt aber nicht den Schluss zu, dass das Gemeinschaftsrecht „pay equity plans“ rundum verbieten würde. Ausgangspunkt der rechtlichen Betrachtung muss auch hier die Überlegung sein, dass ein Verfahrensgesetz anders als der individualrechtliche Anspruch die Entgeltgleichheit im kollektiven Zusammenhang betrachtet.

\section{Verfahrensgesetz als positive Maßnahme?}

Für eine kollektive Betrachtung von Diskriminierungen zeigt sich das Gemeinschaftsrecht durchaus offen. Insbesondere Art. 157 Abs. 4 AEUV erlaubt explizit positive Maßnahmen: „Im Hinblick auf die effektive Gewährleistung der vollen Gleichstellung von Männern und Frauen im Arbeitsleben hindert der Grundsatz der Gleichbehandlung die Mitgliedstaaten nicht daran, zur Erleichterung der Berufstätigkeit des unterrepräsentierten Geschlechts oder zur Verhinderung bzw. zum Ausgleich von Benachteiligungen in der beruflichen Laufbahn spezifische Vergünstigungen beizubehalten oder zu beschließen." 19 Der Europäische Gerichtshof hat in zahlreichen Entscheidungen zu gleichstellungspolitischen Maßnahmen wie Bevorzugungsregelungen für Frauen („Quoten“) bereits entschieden, dass diese Norm das Gleichbehandlungsrecht aus einer formalen und individuellen Betrachtung löst und es auch erlaubt, „kollektiv“ erlittene Benachteiligungen zu berücksichtigen. Akteur(inn)e(n) dürfen nicht nur „negativ“ zur Unterlassung von Diskriminierung angehalten, sondern auch „positiv“ zu „proaktiven“ Maßnahmen verpflichtet werden. ${ }^{20}$

Fallen „pay equity plans“ aber unter diese Erlaubnis? Zunächst kann kaum ein Zweifel daran bestehen, dass ein solches Verfahrensgesetz eine proaktive gesetzgeberische Maßnahme wäre, die einem Ausgleich von Benachteiligungen in der beruflichen Laufbahn diente. Allerdings müsste gewährleistet sein, dass die Maßnahme hierzu wirklich geeignet ist. Diese Prüfung stellt das rechtliche Nadelöhr und gleichzeitig das Einfallstor für eine umfassende rechtliche Überprüfung der verhandelten Pläne dar. Hier wird man sich auch nicht auf einen prozeduralen Ansatz verlassen können. Schon in der Praxis der Regelung aus Ontario hat sich gezeigt, dass eine geschlechtsparitätische Zusammensetzung der Entgeltgleichheitskommissionen ${ }^{21}$ nicht notwendig zu geschlechtergerechteren Regelungen führt. Es wird auch kaum ausreichen können, wenn diese Kommissionen eine Anleitung für die geschlechtsneutrale Bewertung von Tätigkeiten und Arbeitsplätzen erhalten hatten. ${ }^{22}$

Zur Verfahrenskontrolle muss also eine inhaltliche Kontrolle des Endergebnisses kommen ${ }^{23}$, das heißt: Am Ende muss ein geschlechtergerechtes Ergebnis stehen. Dies bedeutet für die Ansprüche nach einem Verfahrensgesetz: Ansprüche für die Zeit vor der Überprüfung richten sich auf Gleichbehandlung im bisherigen System, also auf das Entgelt, das die Bevorzugten erhalten. Ansprüche für die Zeit nach der Überprüfung richten sich auf Gleichbehandlung im neuen System (dem nach Entgeltgleichheitsplan vorgesehenen Endzustand) - soweit dieser diskriminierungsfrei ist. ${ }^{24}$ Und: Ansprüche für die Zeitdauer der Überprüfung und Anpassung richten sich nach dem Entgeltgleichheitsplan, soweit die betreffende Person im neuen Entgeltsystem (dem Endzustand des Plans) nicht mehr diskriminiert ist.

Bei dieser Prüfung ist bisher allerdings eine wichtige Frage außen vor geblieben. Denn bisher wurde die Erlaubnis für positive Maßnahmen nur dafür eingesetzt, Eingriffe in Rechte von „Privilegierten“ zu rechtfertigen; so müssen die Rechte von Männern auf Gleichbehandlung zurücktreten, wenn eine Gleichstellungsmaßnahme verlangt, dass Frauen bei gleicher Qualifikation bevorzugt zu berücksichtigen sind. Kann eine positive Maßnahme aber auch das Zurücktreten von Ansprüchen der Diskriminierten selbst rechtfertigen? Denn die Norm erlaubt ja nur „spezifische Vergünstigungen“.

Andererseits kann man im Erlass des Gesetzes, das den Arbeitgebern bzw. den Tarifparteien zusätzliche belastende Verfahren auferlegt, durchaus eine Vergünstigung sehen. Der Prüfungsmaßstab muss deshalb ein anderer sein als für Übergangsregelungen, die private Akteure aus eigenem Willen heraus vorsehen. ${ }^{25}$ Die bisherige Rechtslage, die auf eine solche Überprüfungspflicht verzichtet, bringt ja gravierende Nachteile für die betroffenen Frauen mit sich, da sie auf das individuelle Vorgehen angewiesen sind. Sie erhalten durch die Verpflichtung zur fall-unabhängigen Kontrolle einen Ausgleich dieser Nachteile.

\section{Tarifautonomie?}

Dies muss jedenfalls dann gelten, wenn der Gesetzgeber im Bereich der Tarifautonomie handelt. Denn das Verfahrensgesetz beließe zwar die tarifpolitischen Einschätzungsprärogativen in der Hand der Tarifparteien; die Auferlegung eines Verhandlungs- und im Streitfall auch eines Schiedsverfahrens stellt jedoch einen Eingriff dar, der gerechtfertigt werden muss. ${ }^{26}$ Auch die Argumente, die eine schrittweise Anpassung sinnvoll erscheinen lassen, beziehen sich auf den kollektiven Aushandlungsprozess - und tatsächlich sind in einem solchen

17 Siehe z.B. Höfer, Besitzstandsfragen bei der Angleichung von betrieblichen Altersrenten an Europarecht, BB 1994, $2139 \mathrm{ff}$.

18 Raasch (Fn. 5).

19 Siehe auch Art. 3 der Richtlinie 2006/54/EG; § 5 AGG.

20 Zu den Begriffen und Ansätzen siehe z.B. Schiek/Schiek (Fn. 7), Einl, Rn. 54 ff.; zur Rechtsprechung des EuGH zu positiven Maßnahmen ebd., $§ 5$, Rn. 5 ff.

21 Kritisch zu solchen geschlechtsstereotypisierenden Ansätzen Kocher, KJ 1999, 182 ff.

22 Dies hat u.a. Winter (Fn. 12), 333 vorgeschlagen. Ähnlich auch $\$ \S 4$ III 12 des Entwurfs der Expertinnenkommission für ein deutsches Gleichstellungsgesetz für die Privatwirtschaft (siehe Fn. 9).

23 Etwa wie von Pfarr vorgeschlagen (Fn. 8).

24 Auf die von Pfarr ebenfalls vorgeschlagene bindende Wirkung einer gerichtlichen (kollektiven?) „Überprüfung der Ergebnisse des Pay Equity Plans" gehe ich hier nicht ein; sie wirft noch einmal andere Probleme auf.

25 EuGH 28.9.1994-C-408/92 (Smith), ArbuR 1994, 429 f.

26 Siehe auch Kocher, Wer schützt die Beschäftigten im transnationalen Standortwettbewerb? Zum Verhältnis von Recht und Politik nach der EuGH-Entscheidung „Viking“, FS Zachert 2010, 37 ff. zur Berücksichtigung der Tarifautonomie in der Rechtskontrolle. 
Prozess tarifpolitische und finanzielle Konsequenzen ganz anderer Art zu berücksichtigen als bei der Befriedigung individueller Rechtsansprüche.

Interessanterweise hatte das BAG sich erst kürzlich mit einer ähnlichen Frage zu beschäftigen, allerdings - wie sollte es anders sein - nicht anlässlich der Geschlechtsdiskriminierung, die selten vor Gericht kommt, sondern anlässlich der Altersdiskriminierung. ${ }^{27}$ Es ging um die Überleitung vom BAT auf den TVöD, also darum, dass im öffentlichen Dienst das bisherige Vergütungssystem, das Lebensaltersstufen und damit $\left(\right.$ wohl $\left.^{28}\right)$ Altersdiskriminierungen enthielt, durch ein neues Vergütungssystem ohne Altersdifferenzierungen abgelöst wurde. Die Überleitung wurde so vollzogen, dass der Besitzstand der Beschäftigten gewahrt wurde; die diskriminierenden Regelungen würden damit zunächst fortwirken, allerdings nur für eine befristete Übergangszeit, da die im alten System erworbenen Rechte im neuen System nicht mehr $1: 1$ abgebildet und sukzessive mit jedem Erreichen einer höheren Stufe oder einer Beförderung abgebaut werden. Auch hier ging es also darum, ob die Tarifvertragsparteien ein gesamtes Entgeltsystem, in dem einzelne Elemente gegen ein Diskriminierungsverbot verstoßen, unverzüglich und vollständig beseitigen müssen oder ob sie schrittweise vorgehen dürfen.

Für einen einzelnen Arbeitgeber hat der EuGH zwar entschieden, dass für die Umstellung einer betrieblichen Altersversorgung auf ein diskriminierungsfreies System keine Übergangsmaßnahmen zulässig sein können. ${ }^{29}$ Hier ging es aber um ein kollektives tarifliches System. Als Argument führt das BAG deshalb in erster Linie die Tarifautonomie an. Sie zwinge dazu zu berücksichtigen, dass einerseits eine sofortige Kürzung von Vergütungen von den Gewerkschaftsmitgliedern nicht akzeptiert worden wäre und andererseits die Arbeitgeberseite strikte Kostenneutralität des neuen Tarifsystems verlangt hatte und deshalb eine „Anpassung nach oben“ nicht akzeptiert hätte.

Der EuGH hat in der Vergangenheit zwar wiederholt den Sozialen Dialog und die Tarifautonomie als Instrument des Diskriminierungsschutzes anerkannt. Er stellte aber bereits im Jahre 1988 anhand eines französischen Gesetzes fest, dass die Tarifautonomie keinesfalls als Rechtfertigung für diskriminierende Strukturen dienen könne. Der französische Gesetzgeber hatte damals die Abschaffung diskriminierender Bedingungen vollständig den Sozialpartnern überlassen, weil er den Weg der Tarifverhandlung für die Anpassung der bestehenden Verhältnisse an den Gleichbehandlungsgrundsatz für am effektivsten hielt. Der EuGH hielt dieses Vorgehen letztlich deshalb für unzulässig, weil die Ineffektivität in der Praxis und damit die Unrichtigkeit der Annahme des Gesetzgebers nachgewiesen war: Als Folge des Gesetzes war nur ein Bruchteil der in Frankreich abgeschlossenen Tarifverträge neu verhandelt worden, oder, in den Worten des Generalanwalts: „Die Folgen der Regelung in der Praxis belegen das Fehlen jeglicher wirksamen staatlichen Durchsetzungsgarantie“. ${ }^{30}$ Der EuGH betonte, dass den Sozialpartnern für die Umsetzung des Gleichbehandlungsgebots zumindest eine Frist hätte gesetzt werden müssen. ${ }^{31}$ Umgekehrt lässt sich dieser seither nicht revidierten Rechtsprechung aber auch entnehmen: Wenn aufgrund klarer Fristen absehbar ist, wann der Zustand der Gleichbehandlung eingehalten ist, steht einer Übergangsregelung wenig entgegen - solange die Mitgliedstaaten das Heft des Handelns in der Hand behalten. ${ }^{32}$

Ein Verfahrensgesetz ginge sogar darüber hinaus, indem es positives Handeln zwingend vorschreibt. Mit der Rechtskontrolle über das Endprodukt würde die Verantwortung auch für die Übergangsphase nur abgegeben, soweit das Ergebnis anzuerkennen ist. Ein Verfahrensgesetz würde damit einen Einschnitt in der Rechtslage markieren, der es rechtfertigte, Übergangsmaßnahmen nicht nur anzuordnen, sondern auch rechtlich abzusichern.
27 BAG, 20.5.2010 - 6 AZR 319/09 (A).

28 Diese Frage legte das BAG in einer Entscheidung vom gleichen Tag dem EuGH vor (BAG, 20.5.2010 - 6 AZR 148/o9 (A)).

29 EuGH 28.9.1994-C-408/92 (Smith), ArbuR 1994, $429 \mathrm{f}$.

30 Schlussanträge des Generalanwalts Sir Gordon Slynn, 21.9.1988 C-312/86.

31 EuGH 25.10.1988 - C-312/86 (Kommission./.Frankreich), NJW 1989, 3086.

32 Siehe auch EuGH, 30.1.1985 - C-143/83 (Kommission./.Dänemark), Slg. 1985, 425 ff.; EuGH 10.7.1986 - C-235/84 (Kommission./.Italien). 